\title{
OSTROWSKI TYPE INEQUALITIES PERTAINING STRONGLY CONVEX FUNCTIONS VIA CONFORMABLE FRACTIONAL INTEGRALS AND THEIR APPLICATIONS
}

\author{
ARTION KASHURI AND ROZANA LIKO
}

\begin{abstract}
In the article, by applied the concept of strongly convex function and one known identity, we establish several Ostrowski type inequalities involving conformable fractional integrals. As applications, some new error estimations for the midpoint formula are provided as well.
\end{abstract}

\section{INTRODUCTION}

The subsequent inequality is known as Ostrowski inequality.

Theorem 1. Let $h: I \longrightarrow \mathbb{R}$ be a mapping differentiable on $I^{\circ}$ and let $a_{1}, a_{2} \in I^{\circ}$ with $a_{1}<a_{2}$. If $\left|h^{\prime}(x)\right| \leq M$ for all $x \in\left[a_{1}, a_{2}\right]$, then

$$
\left|h(x)-\frac{1}{a_{2}-a_{1}} \int_{a_{1}}^{a_{2}} h(x) d x\right| \leq M\left(a_{2}-a_{1}\right)\left[\frac{1}{4}+\frac{\left(x-\frac{a_{1}+a_{2}}{2}\right)^{2}}{\left(a_{2}-a_{1}\right)^{2}}\right], \quad \forall x \in\left[a_{1}, a_{2}\right] .
$$

Ostrowski inequality is playing a very important role in all the fields of mathematics, especially in the theory of approximations. Thus such inequalities were studied extensively by many researches and numerous generalizations, extensions, variants and applications can be found in the literature [[1]-[4],[7]-[13],[15],[16],[18]-[20],[23]-[27],[29],[30]].

Definition 1. [17] Given a function $h:[0, \infty) \longrightarrow \mathbb{R}$. Conformable fractional derivative of $h$ of order $\alpha$ is defined by

$$
\mathrm{D}_{\alpha}(h)(t)=\lim _{\epsilon \rightarrow 0} \frac{h\left(t+\epsilon t^{1-\alpha}\right)-h(t)}{\epsilon},
$$

for all $t>0$ and $\alpha \in(0,1]$. If the conformable fractional derivative of $h$ of order $\alpha$ exists, then we say that $h$ is $\alpha$-differentiable.

Let $h$ be $\alpha$-differentiable in $(0, a)$, and $\lim _{t \rightarrow 0^{+}} h^{\alpha}(t)$ exists, then define

$$
h^{\alpha}(0)=\lim _{t \rightarrow 0^{+}} h^{\alpha}(t)
$$

We will sometimes write $h^{\alpha}(t)$ and $\frac{d_{\alpha}}{d_{\alpha} t}(h)$ for $\mathrm{D}_{\alpha}(h)(t)$, to denote the conformable fractional derivatives of $h$ of order $\alpha$.

2010 Mathematics Subject Classification. 26D15, 26A51, 26A33, 26 A42.

Key words and phrases. Convex function, Ostrowski inequality, Hölder's inequality, Power mean inequality, Conformable integrals, Midpoint formula. 
Theorem 2. [17] Let $\alpha \in(0,1]$ and $\phi, \psi$ be $\alpha$-differentiable at a point $t>0$. Then

i. $\frac{d_{\alpha}}{d_{\alpha} t}\left(t^{n}\right)=n t^{n-\alpha}$, for all $n \in \mathbb{R}$.

ii. $\frac{d_{\alpha}}{d_{\alpha} t}(b)=0$, for all constant functions $\phi(t)=b$.

iii. $\frac{d_{\alpha}}{d_{\alpha} t}\left(a_{1} \phi(t)+a_{2} \psi(t)\right)=a_{1} \frac{d_{\alpha}}{d_{\alpha} t}(\phi(t))+a_{2} \frac{d_{\alpha}}{d_{\alpha} t}(\psi(t))$, for all $a_{1}, a_{2} \in \mathbb{R}$.

iv. $\frac{d_{\alpha}}{d_{\alpha} t}(\phi(t) \psi(t))=\phi(t) \frac{d_{\alpha}}{d_{\alpha} t}(\psi(t))+\psi(t) \frac{d_{\alpha}}{d_{\alpha} t}(\phi(t))$.

v. $\frac{d_{\alpha}}{d_{\alpha} t}\left(\frac{\phi(t)}{\psi(t)}\right)=\frac{\psi(t) \frac{d_{\alpha}}{d_{\alpha} t}(\phi(t))-\phi(t) \frac{d_{\alpha}}{d_{\alpha} t}(\psi(t))}{(\psi(t))^{2}}$.

vi. $\frac{d_{\alpha}}{d_{\alpha} t}((\phi \circ \psi)(t))=\phi^{\prime}(\psi(t)) \frac{d_{\alpha}}{d_{\alpha} t}(\psi(t))$, for $\phi$ differentiable at $\psi(t)$.

If, in addition, the function $\phi$ is differentiable, then

$$
\frac{d_{\alpha}}{d_{\alpha} t}(\phi(t))=t^{1-\alpha} \frac{d}{d t}(\phi(t))
$$

Now, let us recall some basic definitions of various convex functions and conformable fractional integral.

Definition 2. A function $h: I \longrightarrow \mathbb{R}, I \subseteq \mathbb{R}$, is said to be convex on $\mathrm{I}$ if the inequality

$$
h\left(t a_{1}+(1-t) a_{2}\right) \leq t h\left(a_{1}\right)+(1-t) h\left(a_{2}\right)
$$

holds for all $a_{1}, a_{2} \in \mathrm{I}$ and $t \in[0,1]$. Also, we say that $h$ is concave, if the inequality (5) is reversed.

Definition 3. A function $h: I \longrightarrow \mathbb{R}$ is called strongly convex with modulus $c>0$, if

$$
h\left(t a_{1}+(1-t) a_{2}\right) \leq t h\left(a_{1}\right)+(1-t) h\left(a_{2}\right)-c t(1-t)\left(a_{2}-a_{1}\right)^{2}
$$

holds for all $a_{1}, a_{2} \in \mathrm{I}$ and $t \in[0,1]$.

Strongly convex functions play important role in optimization theory and mathematical economics. Many properties and applications of them can be found in the literature $[[6],[14],[21],[22],[28]]$.

Definition 4. [5] (Conformable fractional integral). Let $\alpha \in(0,1)$ and $0 \leq a_{1}<a_{2}$. A function $h:\left[a_{1}, a_{2}\right] \longrightarrow \mathbb{R}$ is $\alpha$-fractional integrable on $\left[a_{1}, a_{2}\right]$ if the integral

$$
\int_{a_{1}}^{a_{2}} h(t) d_{\alpha} t=\int_{a_{1}}^{a_{2}} h(t) t^{\alpha-1} d t
$$

exists and is finite. All $\alpha$-fractional integrable functions on $\left[a_{1}, a_{2}\right]$ is indicated by $\mathrm{L}_{\alpha}^{1}\left(\left[a_{1}, a_{2}\right]\right)$.

Remark 1.

$$
\mathrm{I}_{\alpha}^{a_{1}}(h)(t)=\mathrm{I}_{1}^{a_{1}}\left(t^{\alpha-1} h\right)=\int_{a_{1}}^{x} \frac{h(t)}{t^{1-\alpha}} d t,
$$

where the integral is the usual Riemann improper integral, $\alpha \in(0,1]$.

The main purpose of the article is to find several Ostrowski type inequalities involving conformable fractional integrals using the concept of strongly convex functions and one known identity. At the end of the paper we give some error estimations for the midpoint formula. 


\section{Main Results}

In order to prove our main results we need the following lemma.

Lemma 1. [1] Let $0<\alpha \leq 1,0 \leq a_{1}<a_{2}$ and $h:\left[a_{1}, a_{2}\right] \longrightarrow \mathbb{R}$ be an $\alpha$-fractional differentiable function. Then the identity

$$
\begin{aligned}
& h(x)-\frac{\alpha}{a_{2}^{\alpha}-a_{1}^{\alpha}} \int_{a_{1}}^{a_{2}} h(s) d_{\alpha} s \\
= & \frac{x-a_{1}}{a_{2}^{\alpha}-a_{1}^{\alpha}} \int_{0}^{1}\left(\left((1-t) a_{1}+t x\right)^{2 \alpha-1}-a_{1}^{\alpha}\left((1-t) a_{1}+t x\right)^{\alpha-1}\right) \\
\times & D_{\alpha}(h)\left((1-t) a_{1}+t x\right) t^{1-\alpha} d_{\alpha} t \\
+ & \frac{a_{2}-x}{a_{2}^{\alpha}-a_{1}^{\alpha}} \int_{0}^{1}\left(\left((1-t) a_{2}+t x\right)^{2 \alpha-1}-a_{2}^{\alpha}\left((1-t) a_{2}+t x\right)^{\alpha-1}\right) \\
\times & D_{\alpha}(h)\left((1-t) a_{2}+t x\right) t^{1-\alpha} d_{\alpha} t
\end{aligned}
$$

holds if $D_{\alpha}(h) \in L_{\alpha}^{1}\left(\left[a_{1}, a_{2}\right]\right)$.

Theorem 3. Let $0 \leq a_{1}<a_{2}$ and $h:\left[a_{1}, a_{2}\right] \longrightarrow \mathbb{R}$ be an $\alpha$-fractional differentiable function for $\alpha \in(0,1]$. If $D_{\alpha}(h) \in L_{\alpha}^{1}\left(\left[a_{1}, a_{2}\right]\right)$ and $\left|h^{\prime}(x)\right|$ is strongly convex function with modulus $c>0$, then

$$
\begin{aligned}
& \left|h(x)-\frac{\alpha}{a_{2}^{\alpha}-a_{1}^{\alpha}} \int_{a_{1}}^{a_{2}} h(s) d_{\alpha} s\right| \\
& \quad \leq \frac{x-a_{1}}{a_{2}^{\alpha}-a_{1}^{\alpha}} \Delta_{1}+\frac{a_{2}-x}{a_{2}^{\alpha}-a_{1}^{\alpha}} \Delta_{2}-c \frac{\left(x-a_{1}\right)^{3}}{a_{2}^{\alpha}-a_{1}^{\alpha}} \Delta_{3}-c \frac{\left(a_{2}-x\right)^{3}}{a_{2}^{\alpha}-a_{1}^{\alpha}} \Delta_{4},
\end{aligned}
$$

where

$$
\begin{aligned}
\Delta_{1} & =\frac{1}{6} a_{1}^{\alpha-1} x\left|h^{\prime}\left(a_{1}\right)\right|+\frac{1}{12} x^{\alpha-1} a_{1}\left|h^{\prime}\left(a_{1}\right)\right|+\frac{1}{12} x\left|h^{\prime}\left(a_{1}\right)\right|-\frac{1}{4} a_{1}^{\alpha}\left|h^{\prime}\left(a_{1}\right)\right| \\
& +\frac{1}{12} a_{1}\left|h^{\prime}(x)\right|+\frac{1}{12} x^{\alpha-1} a_{1}\left|h^{\prime}(x)\right|+\frac{1}{4} x\left|h^{\prime}(x)\right|-\frac{1}{2} a_{1}^{\alpha}\left|h^{\prime}(x)\right|, \\
\Delta_{2} & =\frac{1}{6} a_{2}^{\alpha}\left|h^{\prime}\left(a_{2}\right)\right|-\frac{1}{6} x^{\alpha}\left|h^{\prime}\left(a_{2}\right)\right|+\frac{1}{3} a_{2}^{\alpha}\left|h^{\prime}(x)\right|-\frac{1}{3} x^{\alpha}\left|h^{\prime}(x)\right| \\
\Delta_{3} & =\frac{a_{1}^{\alpha}+x^{\alpha}}{20}+\frac{a_{1} x^{\alpha-1}+x a_{1}^{\alpha-1}}{30}-\frac{a_{1}^{\alpha}}{6} \\
\Delta_{4} & =\frac{a_{2}^{\alpha}}{6}-\frac{a_{2}^{\alpha}+x^{\alpha}}{12} .
\end{aligned}
$$

Proof. By Lemma 1, the fact that $x^{\alpha-1}$ and $-x^{\alpha}$ are both convex for $x>0$, properties of the modulus and since the function $\left|h^{\prime}(x)\right|$ is strongly convex with modulus $c>0$, we 
have

$$
\begin{aligned}
& \left|h(x)-\frac{\alpha}{a_{2}^{\alpha}-a_{1}^{\alpha}} \int_{a_{1}}^{a_{2}} h(s) d_{\alpha} s\right| \\
& \leq \frac{x-a_{1}}{a_{2}^{\alpha}-a_{1}^{\alpha}} \int_{0}^{1}\left(\left((1-t) a_{1}+t x\right)^{\alpha}-a_{1}^{\alpha}\right)\left|h^{\prime}\left((1-t) a_{1}+t x\right)\right| d t \\
& +\frac{a_{2}-x}{a_{2}^{\alpha}-a_{1}^{\alpha}} \int_{0}^{1}\left(a_{2}^{\alpha}-\left((1-t) a_{2}+t x\right)^{\alpha}\right)\left|h^{\prime}\left((1-t) a_{2}+t x\right)\right| d t \\
& \leq \frac{x-a_{1}}{a_{2}^{\alpha}-a_{1}^{\alpha}} \int_{0}^{1}\left(\left((1-t) a_{1}+t x\right)^{\alpha-1}\left((1-t) a_{1}+t x\right)-a_{1}^{\alpha}\right)\left|h^{\prime}\left((1-t) a_{1}+t x\right)\right| d t \\
& +\frac{a_{2}-x}{a_{2}^{\alpha}-a_{1}^{\alpha}} \int_{0}^{1}\left(a_{2}^{\alpha}-\left((1-t) a_{2}^{\alpha}+t x^{\alpha}\right)\right)\left|h^{\prime}\left((1-t) a_{2}+t x\right)\right| d t \\
& \leq \frac{x-a_{1}}{a_{2}^{\alpha}-a_{1}^{\alpha}} \int_{0}^{1}\left(\left((1-t) a_{1}^{\alpha-1}+t x^{\alpha-1}\right)\left((1-t) a_{1}+t x\right)-a_{1}^{\alpha}\right)\left|h^{\prime}\left((1-t) a_{1}+t x\right)\right| d t \\
& +\frac{a_{2}-x}{a_{2}^{\alpha}-a_{1}^{\alpha}} \int_{0}^{1}\left(a_{2}^{\alpha}-\left((1-t) a_{2}^{\alpha}+t x^{\alpha}\right)\right)\left|h^{\prime}\left((1-t) a_{2}+t x\right)\right| d t \\
& \leq \frac{x-a_{1}}{a_{2}^{\alpha}-a_{1}^{\alpha}} \int_{0}^{1}\left(\left((1-t) a_{1}^{\alpha-1}+t x^{\alpha-1}\right)\left((1-t) a_{1}+t x\right)-a_{1}^{\alpha}\right) \\
& \times \quad\left[(1-t)\left|h^{\prime}\left(a_{1}\right)\right|+t\left|h^{\prime}(x)\right|-c t(1-t)\left(x-a_{1}\right)^{2}\right] d t \\
& +\frac{a_{2}-x}{a_{2}^{\alpha}-a_{1}^{\alpha}} \int_{0}^{1}\left(a_{2}^{\alpha}-\left((1-t) a_{2}^{\alpha}+t x^{\alpha}\right)\right) \\
& \times \quad\left[(1-t)\left|h^{\prime}\left(a_{2}\right)\right|+t\left|h^{\prime}(x)\right|-c t(1-t)\left(a_{2}-x\right)^{2}\right] d t \\
& =\frac{x-a_{1}}{a_{2}^{\alpha}-a_{1}^{\alpha}} \Delta_{1}+\frac{a_{2}-x}{a_{2}^{\alpha}-a_{1}^{\alpha}} \Delta_{2}-c \frac{\left(x-a_{1}\right)^{3}}{a_{2}^{\alpha}-a_{1}^{\alpha}} \Delta_{3}-c \frac{\left(a_{2}-x\right)^{3}}{a_{2}^{\alpha}-a_{1}^{\alpha}} \Delta_{4} \text {. }
\end{aligned}
$$

Hence, we have the result in (9).

Corollary 1. If we take $c \longrightarrow 0^{+}$in Theorem 3, we obtain (see [1], Theorem 2.2).

Corollary 2. If we take $x=\left(a_{1}+a_{2}\right) / 2$ in Theorem 3, we get 


$$
\begin{aligned}
& \left|h\left(\frac{a_{1}+a_{2}}{2}\right)-\frac{\alpha}{a_{2}^{\alpha}-a_{1}^{\alpha}} \int_{a_{1}}^{a_{2}} h(s) d_{\alpha} s\right| \\
& \quad \leq \frac{a_{2}-a_{1}}{2\left(a_{2}^{\alpha}-a_{1}^{\alpha}\right)}\left[\left(\frac{2 a_{1}^{\alpha-1} a_{2}-10 a_{1}^{\alpha}+a_{1}+a_{2}}{24}\right)\left|h^{\prime}\left(a_{1}\right)\right|+\frac{a_{1}}{12}\left(\frac{a_{1}+a_{2}}{2}\right)^{\alpha-1}\left|h^{\prime}\left(a_{1}\right)\right|\right. \\
& \quad+\left(\frac{5 a_{1}+3 a_{2}-12 a_{1}^{\alpha}}{24}\right)\left|h^{\prime}\left(\frac{a_{1}+a_{2}}{2}\right)\right|+\frac{a_{1}}{12}\left(\frac{a_{1}+a_{2}}{2}\right)^{\alpha-1}\left|h^{\prime}\left(\frac{a_{1}+a_{2}}{2}\right)\right| \\
& \quad+\frac{a_{2}^{\alpha}}{6}\left|h^{\prime}\left(a_{2}\right)\right|-\frac{1}{6}\left(\frac{a_{1}+a_{2}}{2}\right)^{\alpha}\left|h^{\prime}\left(a_{2}\right)\right| \\
& \left.+\frac{a_{2}^{\alpha}}{3}\left|h^{\prime}\left(\frac{a_{1}+a_{2}}{2}\right)\right|-\frac{1}{3}\left(\frac{a_{1}+a_{2}}{2}\right)^{\alpha}\left|h^{\prime}\left(\frac{a_{1}+a_{2}}{2}\right)\right|\right] \\
& \quad-\frac{c}{8} \frac{\left(a_{2}-a_{1}\right)^{3}}{\left(a_{2}^{\alpha}-a_{1}^{\alpha}\right)}\left[\frac{a_{1}^{\alpha}+\left(\frac{a_{1}+a_{2}}{2}\right)^{\alpha}-\frac{a_{2}^{\alpha}+\left(\frac{a_{1}+a_{2}}{2}\right)^{\alpha}}{20}}{12}\right. \\
& \left.\quad+\frac{a_{2}^{\alpha}-a_{1}^{\alpha}}{6}+\frac{a_{1}\left(\frac{a_{1}+a_{2}}{2}\right)^{\alpha-1}+a_{1}^{\alpha-1}\left(\frac{a_{1}+a_{2}}{2}\right)}{30}\right] .
\end{aligned}
$$

Remark 2. If $\alpha=1$, then Corollary 2 becomes

$$
\begin{aligned}
& \left|h\left(\frac{a_{1}+a_{2}}{2}\right)-\frac{1}{a_{2}-a_{1}} \int_{a_{1}}^{a_{2}} h(s) d s\right| \\
& \quad \leq\left(\frac{a_{2}-a_{1}}{24}\right)\left[\left|h^{\prime}\left(a_{1}\right)\right|+4\left|h^{\prime}\left(\frac{a_{1}+a_{2}}{2}\right)\right|+\left|h^{\prime}\left(a_{2}\right)\right|-\frac{c}{4}\left(a_{2}-a_{1}\right)^{2}\right] .
\end{aligned}
$$

Theorem 4. Let $0 \leq a_{1}<a_{2}$ and $h:\left[a_{1}, a_{2}\right] \longrightarrow \mathbb{R}$ be an $\alpha$-fractional differentiable function for $\alpha \in(0,1]$. If $D_{\alpha}(h) \in L_{\alpha}^{1}\left(\left[a_{1}, a_{2}\right]\right)$ and $\left|h^{\prime}(x)\right|^{q}$ is strongly convex function with modulus $c>0$ for $q>1$ and $p^{-1}+q^{-1}=1$, then

$$
\begin{aligned}
& \left|h(x)-\frac{\alpha}{a_{2}^{\alpha}-a_{1}^{\alpha}} \int_{a_{1}}^{a_{2}} h(s) d \alpha s\right| \\
& \quad \leq \frac{x-a_{1}}{a_{2}^{\alpha}-a_{1}^{\alpha}}\left(A_{1}(\alpha, p)\right)^{\frac{1}{p}}\left[\frac{\left|h^{\prime}\left(a_{1}\right)\right|^{q}+\left|h^{\prime}(x)\right|^{q}}{2}-\frac{c}{6}\left(x-a_{1}\right)^{2}\right]^{\frac{1}{q}} \\
& \quad+\frac{a_{2}-x}{a_{2}^{\alpha}-a_{1}^{\alpha}}\left(A_{2}(\alpha, p)\right)^{\frac{1}{p}}\left[\frac{\left|h^{\prime}\left(a_{2}\right)\right|^{q}+\left|h^{\prime}(x)\right|^{q}}{2}-\frac{c}{6}\left(a_{2}-x\right)^{2}\right]^{\frac{1}{q}},
\end{aligned}
$$




$$
\text { where } \begin{aligned}
A_{1}(\alpha, p) & =\int_{0}^{1}\left(\left((1-t) a_{1}+t x\right)^{\alpha}-a_{1}^{\alpha}\right)^{p} d t=\frac{1}{\left(x-a_{1}\right)} \int_{a_{1}}^{x}\left(t^{\alpha}-a_{1}^{\alpha}\right)^{p} d t \\
A_{2}(\alpha, p) & =\int_{0}^{1}\left(a_{2}^{\alpha}-\left((1-t) a_{2}+t x\right)^{\alpha}\right)^{p} d t=\frac{1}{\left(a_{2}-x\right)} \int_{x}^{a_{2}}\left(a_{2}^{\alpha}-t^{\alpha}\right)^{p} d t .
\end{aligned}
$$

Proof. Using Lemma 1, properties of the modulus, Hölder's inequality and since the function $\left|h^{\prime}(x)\right|^{q}$ is strongly convex with modulus $c>0$, we have

$$
\begin{aligned}
& \left|h(x)-\frac{\alpha}{a_{2}^{\alpha}-a_{1}^{\alpha}} \int_{a_{1}}^{a_{2}} h(s) d_{\alpha} s\right| \\
\leq & \frac{x-a_{1}}{a_{2}^{\alpha}-a_{1}^{\alpha}} \int_{0}^{1}\left(\left((1-t) a_{1}+t x\right)^{\alpha}-a_{1}^{\alpha}\right)\left|h^{\prime}\left((1-t) a_{1}+t x\right)\right| d t \\
+ & \frac{a_{2}-x}{a_{2}^{\alpha}-a_{1}^{\alpha}} \int_{0}^{1}\left(a_{2}^{\alpha}-\left((1-t) a_{2}+t x\right)^{\alpha}\right)\left|h^{\prime}\left((1-t) a_{2}+t x\right)\right| d t \\
\leq & \frac{x-a_{1}}{a_{2}^{\alpha}-a_{1}^{\alpha}}\left(\int_{0}^{1}\left(\left((1-t) a_{1}+t x\right)^{\alpha}-a_{1}^{\alpha}\right)^{p} d t\right)^{\frac{1}{p}}\left(\int_{0}^{1}\left|h^{\prime}\left((1-t) a_{1}+t x\right)\right|^{q} d t\right)^{\frac{1}{q}} \\
+ & \frac{a_{2}-x}{a_{2}^{\alpha}-a_{1}^{\alpha}}\left(\int_{0}^{1}\left(a_{2}^{\alpha}-\left((1-t) a_{2}+t x\right)^{\alpha}\right)^{p} d t\right)^{\frac{1}{p}}\left(\int_{0}^{1}\left|h^{\prime}\left((1-t) a_{2}+t x\right)\right|^{q} d t\right)^{\frac{1}{q}} \\
\leq & \frac{x-a_{1}}{a_{2}^{\alpha}-a_{1}^{\alpha}}\left(\mathrm{A}_{1}(\alpha, p)\right)^{\frac{1}{p}}\left(\int_{0}^{1}\left[(1-t)\left|h^{\prime}\left(a_{1}\right)\right|^{q}+t\left|h^{\prime}(x)\right|^{q}-c t(1-t)\left(x-a_{1}\right)^{2}\right] d t\right)^{\frac{1}{q}} \\
+ & \frac{a_{2}-x}{a_{2}^{\alpha}-a_{1}^{\alpha}}\left(\mathrm{A}_{2}(\alpha, p)\right)^{\frac{1}{p}}\left(\int_{0}^{1}\left[(1-t)\left|h^{\prime}\left(a_{2}\right)\right|^{q}+t\left|h^{\prime}(x)\right|^{q}-c t(1-t)\left(a_{2}-x\right)^{2}\right] d t\right)^{\frac{1}{q}} \\
= & \frac{x-a_{1}}{a_{2}^{\alpha}-a_{1}^{\alpha}}\left(\mathrm{A}_{1}(\alpha, p)\right)^{\frac{1}{p}}\left[\frac{\left|h^{\prime}\left(a_{1}\right)\right|^{q}+\left|h^{\prime}(x)\right|^{q}}{2}-\frac{c}{6}\left(x-a_{1}\right)^{2}\right]^{\frac{1}{q}} \\
+ & \frac{a_{2}-x}{a_{2}^{\alpha}-a_{1}^{\alpha}}\left(\mathrm{A}_{2}(\alpha, p)\right)^{\frac{1}{p}}\left[\frac{\left|h^{\prime}\left(a_{2}\right)\right|^{q}+\left|h^{\prime}(x)\right|^{q}}{2}-\frac{c}{6}\left(a_{2}-x\right)^{2}\right]^{\frac{1}{q}} \cdot
\end{aligned}
$$

Hence, we have the result in (10).

Corollary 3. If we take $c \longrightarrow 0^{+}$in Theorem 4, we get the following inequality

$$
\begin{aligned}
& \left|h(x)-\frac{\alpha}{a_{2}^{\alpha}-a_{1}^{\alpha}} \int_{a_{1}}^{a_{2}} h(s) d_{\alpha} s\right| \\
& \quad \leq \frac{x-a_{1}}{a_{2}^{\alpha}-a_{1}^{\alpha}}\left(A_{1}(\alpha, p)\right)^{\frac{1}{p}}\left[\frac{\left|h^{\prime}\left(a_{1}\right)\right|^{q}+\left|h^{\prime}(x)\right|^{q}}{2}\right]^{\frac{1}{q}} \\
& \quad+\frac{a_{2}-x}{a_{2}^{\alpha}-a_{1}^{\alpha}}\left(A_{2}(\alpha, p)\right)^{\frac{1}{p}}\left[\frac{\left|h^{\prime}\left(a_{2}\right)\right|^{q}+\left|h^{\prime}(x)\right|^{q}}{2}\right]^{\frac{1}{q}} .
\end{aligned}
$$


Corollary 4. If we take $x=\left(a_{1}+a_{2}\right) / 2$ in Theorem 4, we get

$$
\begin{gathered}
\left|h\left(\frac{a_{1}+a_{2}}{2}\right)-\frac{\alpha}{a_{2}^{\alpha}-a_{1}^{\alpha}} \int_{a_{1}}^{a_{2}} h(s) d_{\alpha} s\right| \\
\leq \frac{a_{2}-a_{1}}{2\left(a_{2}^{\alpha}-a_{1}^{\alpha}\right)}\left\{\left(B_{1}(\alpha, p)\right)^{\frac{1}{p}}\left[\frac{\left|h^{\prime}\left(a_{1}\right)\right|^{q}+\left|h^{\prime}\left(\frac{a_{1}+a_{2}}{2}\right)\right|^{q}}{2}-\frac{c}{24}\left(a_{2}-a_{1}\right)^{2}\right]^{\frac{1}{q}}\right. \\
\left.+\left(B_{2}(\alpha, p)\right)^{\frac{1}{p}}\left[\frac{\left|h^{\prime}\left(a_{2}\right)\right|^{q}+\left|h^{\prime}\left(\frac{a_{1}+a_{2}}{2}\right)\right|^{q}}{2}-\frac{c}{24}\left(a_{2}-a_{1}\right)^{2}\right]^{\frac{1}{q}}\right\}, \\
\text { where } B_{1}(\alpha, p)=\frac{2}{\left(a_{2}-a_{1}\right)} \int_{a_{1}}^{\frac{a_{1}+a_{2}}{2}}\left(t^{\alpha}-a_{1}^{\alpha}\right)^{p} d t \\
B_{2}(\alpha, p)=\frac{2}{\left(a_{2}-a_{1}\right)} \int_{\frac{a_{1}+a_{2}}{2}}^{a_{2}}\left(a_{2}^{\alpha}-t^{\alpha}\right)^{p} d t .
\end{gathered}
$$

Remark 3. If $\alpha=1$, then Corollary 4 becomes

$$
\begin{aligned}
& \left|h\left(\frac{a_{1}+a_{2}}{2}\right)-\frac{1}{a_{2}-a_{1}} \int_{a_{1}}^{a_{2}} h(s) d s\right| \\
& \quad \leq\left(\frac{a_{2}-a_{1}}{4}\right)\left(\frac{1}{p+1}\right)^{\frac{1}{p}} \times\left\{\left[\frac{\left|h^{\prime}\left(a_{1}\right)\right|^{q}+\left|h^{\prime}\left(\frac{a_{1}+a_{2}}{2}\right)\right|^{q}}{2}-\frac{c}{24}\left(a_{2}-a_{1}\right)^{2}\right]^{\frac{1}{q}}\right. \\
& \left.\quad+\left[\frac{\left|h^{\prime}\left(a_{2}\right)\right|^{q}+\left|h^{\prime}\left(\frac{a_{1}+a_{2}}{2}\right)\right|^{q}}{2}-\frac{c}{24}\left(a_{2}-a_{1}\right)^{2}\right]^{\frac{1}{q}}\right\} .
\end{aligned}
$$

Theorem 5. Let $M>0,0 \leq a_{1}<a_{2}$ and $h:\left[a_{1}, a_{2}\right] \longrightarrow \mathbb{R}$ be an $\alpha$-fractional differentiable function for $\alpha \in(0,1]$. If $D_{\alpha}(h) \in L_{\alpha}^{1}\left(\left[a_{1}, a_{2}\right]\right)$ and $\left|h^{\prime}(x)\right|^{q}$ is strongly convex function with modulus $c>0$ for $q \geq 1$ and $\left|h^{\prime}(x)\right| \leq M, \forall x \in\left[a_{1}, a_{2}\right]$, then

$$
\begin{aligned}
& \left|h(x)-\frac{\alpha}{a_{2}^{\alpha}-a_{1}^{\alpha}} \int_{a_{1}}^{a_{2}} h(s) d_{\alpha} s\right| \\
& \leq M\left(\frac{x-a_{1}}{a_{2}^{\alpha}-a_{1}^{\alpha}}\right)\left(A_{1}(\alpha)\right)^{1-\frac{1}{q}}\left[A_{2}(\alpha)+A_{3}(\alpha)-c\left(x-a_{1}\right)^{2} G_{1}(\alpha)\right]^{\frac{1}{q}} \\
& \quad+M\left(\frac{a_{2}-x}{a_{2}^{\alpha}-a_{1}^{\alpha}}\right)\left(B_{1}(\alpha)\right)^{1-\frac{1}{q}}\left[B_{2}(\alpha)+B_{3}(\alpha)-c\left(a_{2}-x\right)^{2} G_{2}(\alpha)\right]^{\frac{1}{q}},
\end{aligned}
$$




$$
\text { where } \begin{aligned}
A_{1}(\alpha) & =\frac{x^{\alpha+1}-a_{1}^{\alpha+1}}{(\alpha+1)\left(x-a_{1}\right)}-a_{1}^{\alpha}, \\
B_{1}(\alpha) & =a_{2}^{\alpha}-\frac{x^{\alpha+1}-a_{2}^{\alpha+1}}{(\alpha+1)\left(a_{2}-x\right)}, \\
A_{2}(\alpha) & =-\frac{a_{1}^{\alpha+1}}{(\alpha+1)\left(x-a_{1}\right)} \frac{(\alpha+2)\left(x-a_{1}\right)+a_{1}}{(\alpha+2)\left(x-a_{1}\right)}+\frac{x^{\alpha+2}}{(\alpha+1)\left(x-a_{1}\right)^{2}(\alpha+2)}-\frac{a_{1}^{\alpha}}{2}, \\
B_{2}(\alpha) & =\frac{a_{2}^{\alpha}}{2}+\frac{a_{2}^{\alpha+1}}{(\alpha+1)\left(a_{2}-x\right)} \frac{(\alpha+2)\left(a_{2}-x\right)+a_{2}}{(\alpha+2)\left(a_{2}-x\right)}-\frac{x^{\alpha+2}}{(\alpha+1)\left(a_{2}-x\right)^{2}(\alpha+2)}, \\
A_{3}(\alpha) & =\frac{x^{\alpha+1}}{(\alpha+1)\left(x-a_{1}\right)} \frac{(\alpha+2)\left(x-a_{1}\right)-x}{(\alpha+2)\left(x-a_{1}\right)}, \frac{a_{1}^{\alpha+2}}{(\alpha+1)\left(x-a_{1}\right)^{2}(\alpha+2)}, \\
B_{3}(\alpha) & =\frac{a_{2}^{\alpha}}{2}-\frac{x_{2}^{\alpha+1}}{(\alpha+1)\left(a_{2}-x\right)} \frac{(\alpha+2)\left(a_{2}-x\right)-x}{(\alpha+2)\left(a_{2}-x\right)}-\frac{a_{2}^{\alpha+2}}{(\alpha+1)\left(a_{2}-x\right)^{2}(\alpha+2)}, \\
G_{1}(\alpha) & =\frac{1}{\left(x-a_{1}\right)^{3}}\left[\frac{x}{\alpha+2}\left(x^{\alpha+2}-a_{1}^{\alpha+2}\right)-\frac{a_{1} x}{\alpha+1}\left(x^{\alpha+1}-a_{1}^{\alpha+1}\right)\right. \\
& \left.-\frac{1}{\alpha+3}\left(x^{\alpha+3}-a_{1}^{\alpha+3}\right)-\frac{a_{1}}{\alpha+2}\left(x^{\alpha+2}-a_{1}^{\alpha+2}\right)\right]-\frac{a_{1}^{\alpha}}{6}, \\
G_{2}(\alpha) & =\frac{a_{2}^{\alpha}}{6}-\frac{1}{\left(a_{2}-x\right)^{3}}\left[\frac{a_{2}}{\alpha+2}\left(a_{2}^{\alpha+2}-x^{\alpha+2}\right)-\frac{1}{\alpha+3}\left(a_{2}^{\alpha+3}-x^{\alpha+3}\right)\right. \\
& \left.-\frac{a_{2} x}{\alpha+1}\left(a_{2}^{\alpha+1}-x^{\alpha+1}\right)+\frac{x}{\alpha+2}\left(a_{2}^{\alpha+2}-x^{\alpha+2}\right)\right]
\end{aligned}
$$

Proof. Using Lemma 1, properties of the modulus, the well-known power mean inequality, $\left|h^{\prime}(x)\right| \leq M, \forall x \in\left[a_{1}, a_{2}\right]$ and since the function $\left|h^{\prime}(x)\right|^{q}$ is strongly convex with modulus $c>0$, we have 


$$
\begin{aligned}
& \left|h(x)-\frac{\alpha}{a_{2}^{\alpha}-a_{1}^{\alpha}} \int_{a_{1}}^{a_{2}} h(s) d_{\alpha} s\right| \\
& \leq \frac{x-a_{1}}{a_{2}^{\alpha}-a_{1}^{\alpha}} \int_{0}^{1}\left(\left((1-t) a_{1}+t x\right)^{\alpha}-a_{1}^{\alpha}\right)\left|h^{\prime}\left((1-t) a_{1}+t x\right)\right| d t \\
& +\frac{a_{2}-x}{a_{2}^{\alpha}-a_{1}^{\alpha}} \int_{0}^{1}\left(a_{2}^{\alpha}-\left((1-t) a_{2}+t x\right)^{\alpha}\right)\left|h^{\prime}\left((1-t) a_{2}+t x\right)\right| d t \\
& \leq \frac{x-a_{1}}{a_{2}^{\alpha}-a_{1}^{\alpha}}\left(\int_{0}^{1}\left(\left((1-t) a_{1}+t x\right)^{\alpha}-a_{1}^{\alpha}\right) d t\right)^{1-\frac{1}{q}} \\
& \times\left(\int_{0}^{1}\left(\left((1-t) a_{1}+t x\right)^{\alpha}-a_{1}^{\alpha}\right)\left|h^{\prime}\left((1-t) a_{1}+t x\right)\right|^{q} d t\right)^{\frac{1}{q}} \\
& +\frac{a_{2}-x}{a_{2}^{\alpha}-a_{1}^{\alpha}}\left(\int_{0}^{1}\left(a_{2}^{\alpha}-\left((1-t) a_{2}+t x\right)^{\alpha}\right) d t\right)^{1-\frac{1}{q}} \\
& \times\left(\int_{0}^{1}\left(a_{2}^{\alpha}-\left((1-t) a_{2}+t x\right)^{\alpha}\right)\left|h^{\prime}\left((1-t) a_{2}+t x\right)\right|^{q} d t\right)^{\frac{1}{q}} \\
& \leq \frac{x-a_{1}}{a_{2}^{\alpha}-a_{1}^{\alpha}}\left(\mathrm{A}_{1}(\alpha)\right)^{1-\frac{1}{q}}\left[\int_{0}^{1}\left(\left((1-t) a_{1}+t x\right)^{\alpha}-a_{1}^{\alpha}\right)\right. \\
& \left.\times\left[(1-t)\left|h^{\prime}\left(a_{1}\right)\right|^{q}+t\left|h^{\prime}(x)\right|^{q}-c t(1-t)\left(x-a_{1}\right)^{2}\right] d t\right]^{\frac{1}{q}} \\
& +\frac{a_{2}-x}{a_{2}^{\alpha}-a_{1}^{\alpha}}\left(\mathrm{A}_{2}(\alpha)\right)^{1-\frac{1}{q}}\left[\int_{0}^{1}\left(a_{2}^{\alpha}-\left((1-t) a_{2}+t x\right)^{\alpha}\right)\right. \\
& \left.\times \quad\left[(1-t)\left|h^{\prime}\left(a_{2}\right)\right|^{q}+t\left|h^{\prime}(x)\right|^{q}-c t(1-t)\left(a_{2}-x\right)^{2}\right] d t\right]^{\frac{1}{q}} \\
& \leq M\left(\frac{x-a_{1}}{a_{2}^{\alpha}-a_{1}^{\alpha}}\right)\left(\mathrm{A}_{1}(\alpha)\right)^{1-\frac{1}{q}}\left[\mathrm{~A}_{2}(\alpha)+\mathrm{A}_{3}(\alpha)-c\left(x-a_{1}\right)^{2} \mathrm{G}_{1}(\alpha)\right]^{\frac{1}{q}} \\
& +M\left(\frac{a_{2}-x}{a_{2}^{\alpha}-a_{1}^{\alpha}}\right)\left(\mathrm{B}_{1}(\alpha)\right)^{1-\frac{1}{q}}\left[\mathrm{~B}_{2}(\alpha)+\mathrm{B}_{3}(\alpha)-c\left(a_{2}-x\right)^{2} \mathrm{G}_{2}(\alpha)\right]^{\frac{1}{q}} .
\end{aligned}
$$

Hence, we have the result in (11).

Corollary 5. If we take $c \longrightarrow 0^{+}$in Theorem 5, we obtain (see [1], Theorem 2.5).

Corollary 6. If we take $x=\left(a_{1}+a_{2}\right) / 2$ in Theorem 5 , we get 


$$
\begin{aligned}
& \left|h\left(\frac{a_{1}+a_{2}}{2}\right)-\frac{\alpha}{a_{2}^{\alpha}-a_{1}^{\alpha}} \int_{a_{1}}^{a_{2}} h(s) d_{\alpha} s\right| \\
\leq & \frac{M}{2}\left(\frac{a_{2}-a_{1}}{a_{2}^{\alpha}-a_{1}^{\alpha}}\right)\left\{\left(C_{1}(\alpha)\right)^{1-\frac{1}{q}}\left[C_{2}(\alpha)+C_{3}(\alpha)-\frac{c}{4}\left(a_{2}-a_{1}\right)^{2} E_{1}(\alpha)\right]^{\frac{1}{q}}\right. \\
+ & \left.\left(D_{1}(\alpha)\right)^{1-\frac{1}{q}}\left[D_{2}(\alpha)+D_{3}(\alpha)-\frac{c}{4}\left(a_{2}-a_{1}\right)^{2} E_{2}(\alpha)\right]^{\frac{1}{q}}\right\},
\end{aligned}
$$


OSTROWSKI TYPE INEQUALITIES PERTAINING STRONGLY CONVEX FUNCTIONS

11

where $C_{1}(\alpha)=\frac{\left(\frac{a_{1}+a_{2}}{2}\right)^{\alpha+1}-a_{1}^{\alpha+1}}{(\alpha+1)\left(\left(\frac{a_{1}+a_{2}}{2}\right)-a_{1}\right)}-a_{1}^{\alpha}$,

$$
\begin{aligned}
& D_{1}(\alpha)=a_{2}^{\alpha}-\frac{\left(\frac{a_{1}+a_{2}}{2}\right)^{\alpha+1}-a_{2}^{\alpha+1}}{(\alpha+1)\left(a_{2}-\left(\frac{a_{1}+a_{2}}{2}\right)\right)} \text {, } \\
& C_{2}(\alpha)=-\frac{a_{1}^{\alpha+1}}{(\alpha+1)\left(\left(\frac{a_{1}+a_{2}}{2}\right)-a_{1}\right)} \frac{(\alpha+2)\left(\left(\frac{a_{1}+a_{2}}{2}\right)-a_{1}\right)+a_{1}}{(\alpha+2)\left(\left(\frac{a_{1}+a_{2}}{2}\right)-a_{1}\right)} \\
& +\frac{\left(\frac{a_{1}+a_{2}}{2}\right)^{\alpha+2}}{(\alpha+1)\left(\left(\frac{a_{1}+a_{2}}{2}\right)-a_{1}\right)^{2}(\alpha+2)}-\frac{a_{1}^{\alpha}}{2} \text {, } \\
& D_{2}(\alpha)=\frac{a_{2}^{\alpha}}{2}+\frac{a_{2}^{\alpha+1}}{(\alpha+1)\left(a_{2}-\left(\frac{a_{1}+a_{2}}{2}\right)\right)} \frac{(\alpha+2)\left(a_{2}-\left(\frac{a_{1}+a_{2}}{2}\right)\right)+a_{2}}{(\alpha+2)\left(a_{2}-\left(\frac{a_{1}+a_{2}}{2}\right)\right)} \\
& -\frac{\left(\frac{a_{1}+a_{2}}{2}\right)^{\alpha+2}}{(\alpha+1)\left(a_{2}-\left(\frac{a_{1}+a_{2}}{2}\right)\right)^{2}(\alpha+2)} \\
& C_{3}(\alpha)=\frac{\left(\frac{a_{1}+a_{2}}{2}\right)^{\alpha+1}}{(\alpha+1)\left(\left(\frac{a_{1}+a_{2}}{2}\right)-a_{1}\right)} \frac{(\alpha+2)\left(\left(\frac{a_{1}+a_{2}}{2}\right)-a_{1}\right)-\left(\frac{a_{1}+a_{2}}{2}\right)}{(\alpha+2)\left(\left(\frac{a_{1}+a_{2}}{2}\right)-a_{1}\right)} \\
& +\frac{a_{1}^{\alpha+2}}{(\alpha+1)\left(\left(\frac{a_{1}+a_{2}}{2}\right)-a_{1}\right)^{2}(\alpha+2)}-\frac{a_{1}^{\alpha}}{2}, \\
& D_{3}(\alpha)=\frac{a_{2}^{\alpha}}{2}-\frac{\left(\frac{a_{1}+a_{2}}{2}\right)^{\alpha+1}}{(\alpha+1)\left(a_{2}-\left(\frac{a_{1}+a_{2}}{2}\right)\right)} \frac{(\alpha+2)\left(a_{2}-\left(\frac{a_{1}+a_{2}}{2}\right)\right)-\left(\frac{a_{1}+a_{2}}{2}\right)}{(\alpha+2)\left(a_{2}-\left(\frac{a_{1}+a_{2}}{2}\right)\right)} \\
& -\frac{a_{2}^{\alpha+2}}{(\alpha+1)\left(a_{2}-\left(\frac{a_{1}+a_{2}}{2}\right)\right)^{2}(\alpha+2)} \\
& E_{1}(\alpha)=\frac{1}{\left(\left(\frac{a_{1}+a_{2}}{2}\right)-a_{1}\right)^{3}} \\
& \times\left[\frac{\left(\frac{a_{1}+a_{2}}{2}\right)}{\alpha+2}\left(\left(\frac{a_{1}+a_{2}}{2}\right)^{\alpha+2}-a_{1}^{\alpha+2}\right)-\frac{a_{1}\left(\frac{a_{1}+a_{2}}{2}\right)}{\alpha+1}\left(\left(\frac{a_{1}+a_{2}}{2}\right)^{\alpha+1}-a_{1}^{\alpha+1}\right)\right. \\
& \left.-\frac{1}{\alpha+3}\left(\left(\frac{a_{1}+a_{2}}{2}\right)^{\alpha+3}-a_{1}^{\alpha+3}\right)-\frac{a_{1}}{\alpha+2}\left(\left(\frac{a_{1}+a_{2}}{2}\right)^{\alpha+2}-a_{1}^{\alpha+2}\right)\right]-\frac{a_{1}^{\alpha}}{6}, \\
& E_{2}(\alpha)=\frac{a_{2}^{\alpha}}{6}-\frac{1}{\left(a_{2}-\left(\frac{a_{1}+a_{2}}{2}\right)\right)^{3}} \\
& \times\left[\frac{a_{2}}{\alpha+2}\left(a_{2}^{\alpha+2}-\left(\frac{a_{1}+a_{2}}{2}\right)^{\alpha+2}\right)-\frac{1}{\alpha+3}\left(a_{2}^{\alpha+3}-\left(\frac{a_{1}+a_{2}}{2}\right)^{\alpha+3}\right)\right. \\
& \left.-\frac{a_{2}\left(\frac{a_{1}+a_{2}}{2}\right)}{\alpha+1}\left(a_{2}^{\alpha+1}-\left(\frac{a_{1}+a_{2}}{2}\right)^{\alpha+1}\right)+\frac{\left(\frac{a_{1}+a_{2}}{2}\right)}{\alpha+2}\left(a_{2}^{\alpha+2}-\left(\frac{a_{1}+a_{2}}{2}\right)^{\alpha+2}\right)\right] \text {. }
\end{aligned}
$$


Remark 4. If $\alpha=1$, then Corollary 6 becomes

$$
\begin{aligned}
& \left|h\left(\frac{a_{1}+a_{2}}{2}\right)-\frac{1}{a_{2}-a_{1}} \int_{a_{1}}^{a_{2}} h(s) d s\right| \\
& \quad \leq \frac{M}{2}\left\{\left(\mathrm{C}_{1}(1)\right)^{1-\frac{1}{q}}\left[\mathrm{C}_{2}(1)+\mathrm{C}_{3}(1)-\frac{c}{4}\left(a_{2}-a_{1}\right)^{2} \mathrm{E}_{1}(1)\right]^{\frac{1}{q}}\right. \\
& \left.\quad+\left(\mathrm{D}_{1}(1)\right)^{1-\frac{1}{q}}\left[\mathrm{D}_{2}(1)+\mathrm{D}_{3}(1)-\frac{c}{4}\left(a_{2}-a_{1}\right)^{2} \mathrm{E}_{2}(1)\right]^{\frac{1}{q}}\right\}
\end{aligned}
$$


where $\mathrm{C}_{1}(1)=\frac{\left(\frac{a_{1}+a_{2}}{2}\right)^{2}-a_{1}^{2}}{2\left(\left(\frac{a_{1}+a_{2}}{2}\right)-a_{1}\right)}-a_{1}$,

$$
\begin{aligned}
& \mathrm{D}_{1}(1)=a_{2}-\frac{\left(\frac{a_{1}+a_{2}}{2}\right)^{2}-a_{2}^{2}}{2\left(a_{2}-\left(\frac{a_{1}+a_{2}}{2}\right)\right)}, \\
& \mathrm{C}_{2}(1)=-\frac{a_{1}^{2}}{6\left(\left(\frac{a_{1}+a_{2}}{2}\right)-a_{1}\right)} \frac{3\left(\left(\frac{a_{1}+a_{2}}{2}\right)-a_{1}\right)+a_{1}}{\left(\left(\frac{a_{1}+a_{2}}{2}\right)-a_{1}\right)} \\
& +\frac{\left(\frac{a_{1}+a_{2}}{2}\right)^{3}}{6\left(\left(\frac{a_{1}+a_{2}}{2}\right)-a_{1}\right)^{2}}-\frac{a_{1}}{2}, \\
& \mathrm{D}_{2}(1)=\frac{a_{2}}{2}+\frac{a_{2}^{2}}{6\left(a_{2}-\left(\frac{a_{1}+a_{2}}{2}\right)\right)} \frac{3\left(a_{2}-\left(\frac{a_{1}+a_{2}}{2}\right)\right)+a_{2}}{\left(a_{2}-\left(\frac{a_{1}+a_{2}}{2}\right)\right)} \\
& -\frac{\left(\frac{a_{1}+a_{2}}{2}\right)^{3}}{6\left(a_{2}-\left(\frac{a_{1}+a_{2}}{2}\right)\right)^{2}} \\
& \mathrm{C}_{3}(1)=\frac{\left(\frac{a_{1}+a_{2}}{2}\right)^{2}}{6\left(\left(\frac{a_{1}+a_{2}}{2}\right)-a_{1}\right)} \frac{3\left(\left(\frac{a_{1}+a_{2}}{2}\right)-a_{1}\right)-\left(\frac{a_{1}+a_{2}}{2}\right)}{\left(\left(\frac{a_{1}+a_{2}}{2}\right)-a_{1}\right)} \\
& +\frac{a_{1}^{3}}{6\left(\left(\frac{a_{1}+a_{2}}{2}\right)-a_{1}\right)^{2}}-\frac{a_{1}}{2}, \\
& \mathrm{D}_{3}(1)=\frac{a_{2}}{2}-\frac{\left(\frac{a_{1}+a_{2}}{2}\right)^{2}}{6\left(a_{2}-\left(\frac{a_{1}+a_{2}}{2}\right)\right)} \frac{3\left(a_{2}-\left(\frac{a_{1}+a_{2}}{2}\right)\right)-\left(\frac{a_{1}+a_{2}}{2}\right)}{\left(a_{2}-\left(\frac{a_{1}+a_{2}}{2}\right)\right)} \\
& -\frac{a_{2}^{3}}{6\left(a_{2}-\left(\frac{a_{1}+a_{2}}{2}\right)\right)^{2}} \text {, } \\
& \mathrm{E}_{1}(1)=\frac{1}{\left(\left(\frac{a_{1}+a_{2}}{2}\right)-a_{1}\right)^{3}} \\
& \times \quad\left[\frac{\left(\frac{a_{1}+a_{2}}{2}\right)}{3}\left(\left(\frac{a_{1}+a_{2}}{2}\right)^{3}-a_{1}^{3}\right)-\frac{a_{1}\left(\frac{a_{1}+a_{2}}{2}\right)}{2}\left(\left(\frac{a_{1}+a_{2}}{2}\right)^{2}-a_{1}^{2}\right)\right. \\
& \left.-\frac{1}{4}\left(\left(\frac{a_{1}+a_{2}}{2}\right)^{4}-a_{1}^{4}\right)-\frac{a_{1}}{3}\left(\left(\frac{a_{1}+a_{2}}{2}\right)^{3}-a_{1}^{3}\right)\right]-\frac{a_{1}}{6}, \\
& \mathrm{E}_{2}(1)=\frac{a_{2}}{6}-\frac{1}{\left(a_{2}-\left(\frac{a_{1}+a_{2}}{2}\right)\right)^{3}} \\
& \times\left[\frac{a_{2}}{3}\left(a_{2}^{3}-\left(\frac{a_{1}+a_{2}}{2}\right)^{3}\right)-\frac{1}{4}\left(a_{2}^{4}-\left(\frac{a_{1}+a_{2}}{2}\right)^{4}\right)\right. \\
& \left.-\frac{a_{2}\left(\frac{a_{1}+a_{2}}{2}\right)}{2}\left(a_{2}^{2}-\left(\frac{a_{1}+a_{2}}{2}\right)^{2}\right)+\frac{\left(\frac{a_{1}+a_{2}}{2}\right)}{3}\left(a_{2}^{3}-\left(\frac{a_{1}+a_{2}}{2}\right)^{3}\right)\right] \text {. }
\end{aligned}
$$


Theorem 6. Let $0 \leq a_{1}<a_{2}$ and $h:\left[a_{1}, a_{2}\right] \longrightarrow \mathbb{R}$ be an $\alpha$-fractional differentiable function for $\alpha \in(0,1]$. If $D_{\alpha}(h) \in L_{\alpha}^{1}\left(\left[a_{1}, a_{2}\right]\right)$ and $\left|h^{\prime}(x)\right|^{q}$ is strongly convex function with modulus $c>0$ and $q \geq 1$, then

$$
\begin{aligned}
& \left|h(x)-\frac{\alpha}{a_{2}^{\alpha}-a_{1}^{\alpha}} \int_{a_{1}}^{a_{2}} h(s) d_{\alpha} s\right| \\
& \quad \leq \frac{x-a_{1}}{a_{2}^{\alpha}-a_{1}^{\alpha}}\left(E_{1}(\alpha)\right)^{1-\frac{1}{q}}\left[\left|h^{\prime}\left(a_{1}\right)\right|^{q} F_{1}(\alpha)+\left|h^{\prime}(x)\right|^{q} H_{1}(\alpha)-c\left(x-a_{1}\right)^{2} \Delta_{3}\right]^{\frac{1}{q}} \\
& \quad+\frac{a_{2}-x}{a_{2}^{\alpha}-a_{1}^{\alpha}}\left(E_{2}(\alpha)\right)^{1-\frac{1}{q}}\left[\left|h^{\prime}\left(a_{2}\right)\right|^{q} F_{2}(\alpha)+\left|h^{\prime}(x)\right|^{q} H_{2}(\alpha)-c\left(a_{2}-x\right)^{2} \Delta_{4}\right]^{\frac{1}{q}},
\end{aligned}
$$

$$
\text { where } \begin{aligned}
E_{1}(\alpha) & =\int_{0}^{1}\left(\left((1-t) a_{1}^{\alpha-1}+t x^{\alpha-1}\right)\left((1-t) a_{1}+t x\right)-a_{1}^{\alpha}\right) d t \\
& =\frac{x^{\alpha}+a_{1}^{\alpha}}{3}+\frac{a_{1} x^{\alpha-1}+x a_{1}^{\alpha-1}}{6}-a_{1}^{\alpha} \\
E_{2}(\alpha) & =\int_{0}^{1}\left(a_{2}^{\alpha}-\left((1-t) a_{2}^{\alpha}+t x^{\alpha}\right)\right) d t=\frac{a_{2}^{\alpha}-x^{\alpha}}{2} \\
F_{1}(\alpha) & =\frac{x^{\alpha}-15 a_{1}^{\alpha}+a_{1} x^{\alpha-1}+x a_{1}^{\alpha-1}}{12} \\
H_{1}(\alpha) & =\frac{3 x^{\alpha}-5 a_{1}^{\alpha}+a_{1} x^{\alpha-1}+x a_{1}^{\alpha-1}}{12} \\
F_{2}(\alpha) & =\frac{a_{2}^{\alpha}-x^{\alpha}}{6} \\
H_{2}(\alpha) & =\frac{a_{2}^{\alpha}-x^{\alpha}}{3} .
\end{aligned}
$$

and $\Delta_{3}, \Delta_{4}$ are defined as in Theorem 3. 
Proof. Using Lemma 1, properties of the modulus, the well-known power mean inequality and since the function $\left|h^{\prime}(x)\right|^{q}$ is strongly convex with modulus $c>0$, we have

$$
\begin{aligned}
& \left|h(x)-\frac{\alpha}{a_{2}^{\alpha}-a_{1}^{\alpha}} \int_{a_{1}}^{a_{2}} h(s) d_{\alpha} s\right| \\
& \leq \frac{x-a_{1}}{a_{2}^{\alpha}-a_{1}^{\alpha}} \int_{0}^{1}\left(\left((1-t) a_{1}+t x\right)^{\alpha}-a_{1}^{\alpha}\right)\left|h^{\prime}\left((1-t) a_{1}+t x\right)\right| d t \\
& +\frac{a_{2}-x}{a_{2}^{\alpha}-a_{1}^{\alpha}} \int_{0}^{1}\left(a_{2}^{\alpha}-\left((1-t) a_{2}+t x\right)^{\alpha}\right)\left|h^{\prime}\left((1-t) a_{2}+t x\right)\right| d t \\
& \leq \frac{x-a_{1}}{a_{2}^{\alpha}-a_{1}^{\alpha}} \int_{0}^{1}\left(\left((1-t) a_{1}+t x\right)^{\alpha-1}\left((1-t) a_{1}+t x\right)-a_{1}^{\alpha}\right)\left|h^{\prime}\left((1-t) a_{1}+t x\right)\right| d t \\
& +\frac{a_{2}-x}{a_{2}^{\alpha}-a_{1}^{\alpha}} \int_{0}^{1}\left(a_{2}^{\alpha}-\left((1-t) a_{2}^{\alpha}+t x^{\alpha}\right)\right)\left|h^{\prime}\left((1-t) a_{2}+t x\right)\right| d t \\
& \leq \frac{x-a_{1}}{a_{2}^{\alpha}-a_{1}^{\alpha}} \int_{0}^{1}\left(\left((1-t) a_{1}^{\alpha-1}+t x^{\alpha-1}\right)\left((1-t) a_{1}+t x\right)-a_{1}^{\alpha}\right)\left|h^{\prime}\left((1-t) a_{1}+t x\right)\right| d t \\
& +\frac{a_{2}-x}{a_{2}^{\alpha}-a_{1}^{\alpha}} \int_{0}^{1}\left(a_{2}^{\alpha}-\left((1-t) a_{2}^{\alpha}+t x^{\alpha}\right)\right)\left|h^{\prime}\left((1-t) a_{2}+t x\right)\right| d t \\
& \leq \frac{x-a_{1}}{a_{2}^{\alpha}-a_{1}^{\alpha}}\left(\int_{0}^{1}\left(\left((1-t) a_{1}^{\alpha-1}+t x^{\alpha-1}\right)\left((1-t) a_{1}+t x\right)-a_{1}^{\alpha}\right) d t\right)^{1-\frac{1}{q}} \\
& \times\left(\int_{0}^{1}\left(\left((1-t) a_{1}^{\alpha-1}+t x^{\alpha-1}\right)\left((1-t) a_{1}+t x\right)-a_{1}^{\alpha}\right)\left|h^{\prime}\left((1-t) a_{1}+t x\right)\right|^{q} d t\right)^{\frac{1}{q}} \\
& +\frac{a_{2}-x}{a_{2}^{\alpha}-a_{1}^{\alpha}}\left(\int_{0}^{1}\left(a_{2}^{\alpha}-\left((1-t) a_{2}^{\alpha}+t x^{\alpha}\right)\right) d t\right)^{1-\frac{1}{q}} \\
& \times\left(\int_{0}^{1}\left(a_{2}^{\alpha}-\left((1-t) a_{2}^{\alpha}+t x^{\alpha}\right)\right)\left|h^{\prime}\left((1-t) a_{2}+t x\right)\right|^{q} d t\right)^{\frac{1}{q}} \\
& \leq \frac{x-a_{1}}{a_{2}^{\alpha}-a_{1}^{\alpha}}\left(\mathrm{E}_{1}(\alpha)\right)^{1-\frac{1}{q}}\left[\int_{0}^{1}\left(\left((1-t) a_{1}^{\alpha-1}+t x^{\alpha-1}\right)\left((1-t) a_{1}+t x\right)-a_{1}^{\alpha}\right)\right. \\
& \left.\times \quad\left[(1-t)\left|h^{\prime}\left(a_{1}\right)\right|^{q}+t\left|h^{\prime}(x)\right|^{q}-c t(1-t)\left(x-a_{1}\right)^{2}\right] d t\right]^{\frac{1}{q}} \\
& +\frac{a_{2}-x}{a_{2}^{\alpha}-a_{1}^{\alpha}}\left(\mathrm{E}_{2}(\alpha)\right)^{1-\frac{1}{q}}\left[\int_{0}^{1}\left(a_{2}^{\alpha}-\left((1-t) a_{2}^{\alpha}+t x^{\alpha}\right)\right)\right. \\
& \left.\times\left[(1-t)\left|h^{\prime}\left(a_{2}\right)\right|^{q}+t\left|h^{\prime}(x)\right|^{q}-c t(1-t)\left(a_{2}-x\right)^{2}\right] d t\right]^{\frac{1}{q}} \\
& =\frac{x-a_{1}}{a_{2}^{\alpha}-a_{1}^{\alpha}}\left(\mathrm{E}_{1}(\alpha)\right)^{1-\frac{1}{q}}\left[\left|h^{\prime}\left(a_{1}\right)\right|^{q} \mathrm{~F}_{1}(\alpha)+\left|h^{\prime}(x)\right|{ }^{q} \mathrm{H}_{1}(\alpha)-c\left(x-a_{1}\right)^{2} \Delta_{3}\right]^{\frac{1}{q}} \\
& +\frac{a_{2}-x}{a_{2}^{\alpha}-a_{1}^{\alpha}}\left(\mathrm{E}_{2}(\alpha)\right)^{1-\frac{1}{q}}\left[\left|h^{\prime}\left(a_{2}\right)\right|^{q} \mathrm{~F}_{2}(\alpha)+\left|h^{\prime}(x)\right|^{q} \mathrm{H}_{2}(\alpha)-c\left(a_{2}-x\right)^{2} \Delta_{4}\right]^{\frac{1}{q}} .
\end{aligned}
$$


Hence, we have the result in (12).

Corollary 7. If we take $q=1$ in Theorem 6, we obtain Theorem 3.

Corollary 8. If we take $c \longrightarrow 0^{+}$in Theorem 6 , we get the following inequality

$$
\begin{aligned}
& \left|h(x)-\frac{\alpha}{a_{2}^{\alpha}-a_{1}^{\alpha}} \int_{a_{1}}^{a_{2}} h(s) d_{\alpha} s\right| \\
& \quad \leq \frac{x-a_{1}}{a_{2}^{\alpha}-a_{1}^{\alpha}}\left(E_{1}(\alpha)\right)^{1-\frac{1}{q}}\left[\left|h^{\prime}\left(a_{1}\right)\right|^{q} F_{1}(\alpha)+\left|h^{\prime}(x)\right|^{q} H_{1}(\alpha)\right]^{\frac{1}{q}} \\
& \quad+\frac{a_{2}-x}{a_{2}^{\alpha}-a_{1}^{\alpha}}\left(E_{2}(\alpha)\right)^{1-\frac{1}{q}}\left[\left|h^{\prime}\left(a_{2}\right)\right|{ }^{q} F_{2}(\alpha)+\left|h^{\prime}(x)\right|{ }^{q} H_{2}(\alpha)\right]^{\frac{1}{q}} .
\end{aligned}
$$

Corollary 9. If we take $x=\left(a_{1}+a_{2}\right) / 2$ in Theorem 6 , we get

$$
\begin{aligned}
& \left|h\left(\frac{a_{1}+a_{2}}{2}\right)-\frac{\alpha}{a_{2}^{\alpha}-a_{1}^{\alpha}} \int_{a_{1}}^{a_{2}} h(s) d_{\alpha} s\right| \\
& \leq \frac{a_{2}-a_{1}}{2\left(a_{2}^{\alpha}-a_{1}^{\alpha}\right)}\left\{\left(L_{1}(\alpha)\right)^{1-\frac{1}{q}}\left[\left|h^{\prime}\left(a_{1}\right)\right|^{q} M_{1}(\alpha)+\left|h^{\prime}\left(\frac{a_{1}+a_{2}}{2}\right)\right|^{q} N_{1}(\alpha)-\frac{c}{4}\left(a_{2}-a_{1}\right)^{2} P_{1}(\alpha)\right]^{\frac{1}{q}}\right. \\
& \left.+\quad\left(L_{2}(\alpha)\right)^{1-\frac{1}{q}}\left[\left|h^{\prime}\left(a_{2}\right)\right|^{q} M_{2}(\alpha)+\left|h^{\prime}\left(\frac{a_{1}+a_{2}}{2}\right)\right|^{q} N_{2}(\alpha)-\frac{c}{4}\left(a_{2}-a_{1}\right)^{2} P_{2}(\alpha)\right]^{\frac{1}{q}}\right\}, \\
& \text { where } L_{1}(\alpha)=\frac{\left(\frac{a_{1}+a_{2}}{2}\right)^{\alpha}+a_{1}^{\alpha}}{3}+\frac{a_{1}\left(\frac{a_{1}+a_{2}}{2}\right)^{\alpha-1}+\left(\frac{a_{1}+a_{2}}{2}\right) a_{1}^{\alpha-1}}{6}-a_{1}^{\alpha} \text {, } \\
& L_{2}(\alpha)==\frac{a_{2}^{\alpha}-\left(\frac{a_{1}+a_{2}}{2}\right)^{\alpha}}{2}, \\
& M_{1}(\alpha)=\frac{\left(\frac{a_{1}+a_{2}}{2}\right)^{\alpha}-15 a_{1}^{\alpha}+a_{1}\left(\frac{a_{1}+a_{2}}{2}\right)^{\alpha-1}+\left(\frac{a_{1}+a_{2}}{2}\right) a_{1}^{\alpha-1}}{12}, \\
& N_{1}(\alpha)=\frac{3\left(\frac{a_{1}+a_{2}}{2}\right)^{\alpha}-5 a_{1}^{\alpha}+a_{1}\left(\frac{a_{1}+a_{2}}{2}\right)^{\alpha-1}+\left(\frac{a_{1}+a_{2}}{2}\right) a_{1}^{\alpha-1}}{12}, \\
& M_{2}(\alpha)=\frac{a_{2}^{\alpha}-\left(\frac{a_{1}+a_{2}}{2}\right)^{\alpha}}{6}, \\
& N_{2}(\alpha)=\frac{a_{2}^{\alpha}-\left(\frac{a_{1}+a_{2}}{2}\right)^{\alpha}}{3}, \\
& P_{1}(\alpha)=\frac{a_{1}^{\alpha}+\left(\frac{a_{1}+a_{2}}{2}\right)^{\alpha}}{20}+\frac{a_{1}\left(\frac{a_{1}+a_{2}}{2}\right)^{\alpha-1}+\left(\frac{a_{1}+a_{2}}{2}\right) a_{1}^{\alpha-1}}{30}-\frac{a_{1}^{\alpha}}{6}, \\
& P_{2}(\alpha)=\frac{a_{2}^{\alpha}}{6}-\frac{a_{2}^{\alpha}+\left(\frac{a_{1}+a_{2}}{2}\right)^{\alpha}}{12} .
\end{aligned}
$$

Remark 5. If $\alpha=1$, then Corollary 9 becomes 


$$
\begin{aligned}
& \left|h\left(\frac{a_{1}+a_{2}}{2}\right)-\frac{1}{a_{2}-a_{1}} \int_{a_{1}}^{a_{2}} h(s) d s\right| \\
& \leq \frac{1}{2}\left\{\left(\mathrm{~L}_{1}(1)\right)^{1-\frac{1}{q}}\left[\left|h^{\prime}\left(a_{1}\right)\right|^{q} \mathrm{M}_{1}(1)+\left|h^{\prime}\left(\frac{a_{1}+a_{2}}{2}\right)\right|^{q} \mathrm{~N}_{1}(1)-\frac{c}{4}\left(a_{2}-a_{1}\right)^{2} \mathrm{P}_{1}(1)\right]^{\frac{1}{q}}\right. \\
& +\quad\left(\mathrm{L}_{2}(1)\right)^{1-\frac{1}{q}}\left[\left|h^{\prime}\left(a_{2}\right)\right|{ }^{q} \mathrm{M}_{2}(1)+\left.\left|h^{\prime}\left(\frac{a_{1}+a_{2}}{2}\right)\right|\right|^{q} \mathrm{~N}_{2}(1)-\left.\frac{c}{4}\left(a_{2}-a_{1}\right)^{2} \mathrm{P}_{2}(1)\right|^{\frac{1}{q}}\right\} \\
& \text { where } \mathrm{L}_{1}(1)=\frac{\left(\frac{a_{1}+a_{2}}{2}\right)+a_{1}}{3}+\frac{a_{1}+\left(\frac{a_{1}+a_{2}}{2}\right)}{6}-a_{1} \text {, } \\
& \mathrm{L}_{2}(1)==\frac{a_{2}-\left(\frac{a_{1}+a_{2}}{2}\right)}{2}, \\
& \mathrm{M}_{1}(1)=\frac{\left(\frac{a_{1}+a_{2}}{2}\right)-15 a_{1}+a_{1}+\left(\frac{a_{1}+a_{2}}{2}\right)}{12} \text {, } \\
& \mathrm{N}_{1}(1)=\frac{3\left(\frac{a_{1}+a_{2}}{2}\right)-5 a_{1}+a_{1}+\left(\frac{a_{1}+a_{2}}{2}\right)}{12}, \\
& \mathrm{M}_{2}(1)=\frac{a_{2}-\left(\frac{a_{1}+a_{2}}{2}\right)}{6}, \\
& \mathrm{~N}_{2}(1)=\frac{a_{2}-\left(\frac{a_{1}+a_{2}}{2}\right)}{3}, \\
& \mathrm{P}_{1}(1)=\frac{a_{1}+\left(\frac{a_{1}+a_{2}}{2}\right)}{20}+\frac{a_{1}+\left(\frac{a_{1}+a_{2}}{2}\right)}{30}-\frac{a_{1}}{6}, \\
& \mathrm{P}_{2}(1)=\frac{a_{2}}{6}-\frac{a_{2}+\left(\frac{a_{1}+a_{2}}{2}\right)}{12} .
\end{aligned}
$$

\section{Applications to midpoint formula}

Let $\mathrm{P}$ be the partition of the points $a_{1}=x_{0}<x_{1}<\ldots<x_{n-1}<x_{n}=a_{2}$ of the interval $\left[a_{1}, a_{2}\right]$ and consider the quadrature formula

$$
\int_{a_{1}}^{a_{2}} h(x) d_{\alpha} x=\mathrm{T}_{\alpha}(h, \mathrm{P})+\mathrm{E}_{\alpha}(h, \mathrm{P}),
$$

where

$$
\mathrm{T}_{\alpha}(h, \mathrm{P})=\sum_{i=0}^{n-1} h\left(\frac{x_{i}+x_{i+1}}{2}\right) \frac{\left(x_{i+1}^{\alpha}-x_{i}^{\alpha}\right)}{\alpha}
$$

is the midpoint version and $\mathrm{E}_{\alpha}(h, \mathrm{P})$ denotes the associated approximation error. Here, we are going to derive some new estimates for the midpoint formula. 
Proposition 1. Let $0 \leq x_{0}<x_{n}$ and $h:\left[x_{0}, x_{n}\right] \longrightarrow \mathbb{R}$ be an $\alpha$-fractional differentiable function for $\alpha \in(0,1]$. If $D_{\alpha}(h) \in L_{\alpha}^{1}\left(\left[x_{0}, x_{n}\right]\right)$ and $\left|h^{\prime}(x)\right|$ is strongly convex function with modulus $c>0$, then

$$
\begin{aligned}
\left|E_{\alpha}(h, P)\right| & \leq \sum_{i=0}^{n-1} \frac{\left(x_{i+1}-x_{i}\right)}{2 \alpha}\left[\left(\frac{2 x_{i}^{\alpha-1} x_{i+1}-10 x_{i}^{\alpha}+x_{i}+x_{i+1}}{24}\right)\left|h^{\prime}\left(x_{i}\right)\right|\right. \\
& +\frac{x_{i}}{12}\left(\frac{x_{i}+x_{i+1}}{2}\right)^{\alpha-1}\left|h^{\prime}\left(x_{i}\right)\right|+\left(\frac{5 x_{i}+3 x_{i+1}-12 x_{i}^{\alpha}}{24}\right)\left|h^{\prime}\left(\frac{x_{i}+x_{i+1}}{2}\right)\right| \\
& +\frac{x_{i}}{12}\left(\frac{x_{i}+x_{i+1}}{2}\right)^{\alpha-1}\left|h^{\prime}\left(\frac{x_{i}+x_{i+1}}{2}\right)\right|+\frac{x_{i+1}^{\alpha}}{6}\left|h^{\prime}\left(x_{i+1}\right)\right|-\frac{1}{6}\left(\frac{x_{i}+x_{i+1}}{2}\right)^{\alpha}\left|h^{\prime}\left(x_{i+1}\right)\right| \\
& \left.+\frac{x_{i+1}^{\alpha}}{3}\left|h^{\prime}\left(\frac{x_{i}+x_{i+1}}{2}\right)\right|-\frac{1}{3}\left(\frac{x_{i}+x_{i+1}}{2}\right)^{\alpha}\left|h^{\prime}\left(\frac{x_{i}+x_{i+1}}{2}\right)\right|\right] \\
& -\frac{c}{8 \alpha}\left(x_{i+1}-x_{i}\right)^{3}\left[\frac{\left.x_{i}^{\alpha}+\left(\frac{x_{i}+x_{i+1}}{2}\right)^{\alpha}-\frac{x_{i+1}^{\alpha}+\left(\frac{x_{i}+x_{i+1}}{2}\right)^{\alpha}}{20}\right] .}{30}\right. \\
& +\frac{x_{i+1}^{\alpha}-x_{i}^{\alpha}}{6}+\frac{x_{i}\left(\frac{x_{i}+x_{i+1}}{2}\right)^{\alpha-1}+x_{i}^{\alpha-1}\left(\frac{x_{i}+x_{i+1}}{2}\right)}{30}
\end{aligned}
$$

Proof. Applying Corollary 2 of Theorem 3 on the subintervals $\left[x_{i}, x_{i+1}\right](i=0,1, \ldots, n-1)$ of the partition $\mathrm{P}$, we have

$$
\begin{aligned}
& \left|h\left(\frac{x_{i}+x_{i+1}}{2}\right) \frac{\left(x_{i+1}^{\alpha}-x_{i}^{\alpha}\right)}{\alpha}-\int_{x_{i}}^{x_{i+1}} h(x) d_{\alpha} x\right| \\
\leq & \frac{\left(x_{i+1}-x_{i}\right)}{2 \alpha}\left[\left(\frac{2 x_{i}^{\alpha-1} x_{i+1}-10 x_{i}^{\alpha}+x_{i}+x_{i+1}}{24}\right)\left|h^{\prime}\left(x_{i}\right)\right|+\frac{x_{i}}{12}\left(\frac{x_{i}+x_{i+1}}{2}\right)^{\alpha-1}\left|h^{\prime}\left(x_{i}\right)\right|\right. \\
+ & \left(\frac{5 x_{i}+3 x_{i+1}-12 x_{i}^{\alpha}}{24}\right)\left|h^{\prime}\left(\frac{x_{i}+x_{i+1}}{2}\right)\right|+\frac{x_{i}}{12}\left(\frac{x_{i}+x_{i+1}}{2}\right)^{\alpha-1}\left|h^{\prime}\left(\frac{x_{i}+x_{i+1}}{2}\right)\right| \\
+ & \frac{x_{i+1}^{\alpha}}{6}\left|h^{\prime}\left(x_{i+1}\right)\right|-\frac{1}{6}\left(\frac{x_{i}+x_{i+1}}{2}\right)^{\alpha}\left|h^{\prime}\left(x_{i+1}\right)\right| \\
+ & \left.\frac{x_{i+1}^{\alpha}}{3}\left|h^{\prime}\left(\frac{x_{i}+x_{i+1}}{2}\right)\right|-\frac{1}{3}\left(\frac{x_{i}+x_{i+1}}{2}\right)^{\alpha}\left|h^{\prime}\left(\frac{x_{i}+x_{i+1}}{2}\right)\right|\right] \\
- & \frac{c}{8 \alpha}\left(x_{i+1}-x_{i}\right)^{3}\left[\frac{x_{i}^{\alpha}+\left(\frac{x_{i}+x_{i+1}}{2}\right)^{\alpha}-\frac{x_{i+1}^{\alpha}+\left(\frac{x_{i}+x_{i+1}}{2}\right)^{\alpha}}{20}}{12}\right] . \\
+ & \left.\frac{x_{i+1}^{\alpha}-x_{i}^{\alpha}}{6}+\frac{x_{i}\left(\frac{x_{i}+x_{i+1}}{2}\right)^{\alpha-1}+x_{i}^{\alpha-1}\left(\frac{x_{i}+x_{i+1}}{2}\right)}{30}\right]
\end{aligned}
$$


Hence from above

$$
\begin{aligned}
& \left|\mathrm{E}_{\alpha}(h, \mathrm{P})\right|=\left|\sum_{i=0}^{n-1}\left\{\int_{x_{i}}^{x_{i+1}} h(x) d_{\alpha} x-h\left(\frac{x_{i}+x_{i+1}}{2}\right) \frac{\left(x_{i+1}^{\alpha}-x_{i}^{\alpha}\right)}{\alpha}\right\}\right| \\
\leq & \sum_{i=0}^{n-1}\left|\int_{x_{i}}^{x_{i+1}} h(x) d_{\alpha} x-h\left(\frac{x_{i}+x_{i+1}}{2}\right) \frac{\left(x_{i+1}^{\alpha}-x_{i}^{\alpha}\right)}{\alpha}\right| \\
\leq & \sum_{i=0}^{n-1} \frac{\left(x_{i+1}-x_{i}\right)}{2 \alpha}\left[\left(\frac{2 x_{i}^{\alpha-1} x_{i+1}-10 x_{i}^{\alpha}+x_{i}+x_{i+1}}{24}\right)\left|h^{\prime}\left(x_{i}\right)\right|+\frac{x_{i}}{12}\left(\frac{x_{i}+x_{i+1}}{2}\right)^{\alpha-1}\left|h^{\prime}\left(x_{i}\right)\right|\right. \\
+ & \left(\frac{5 x_{i}+3 x_{i+1}-12 x_{i}^{\alpha}}{24}\right)\left|h^{\prime}\left(\frac{x_{i}+x_{i+1}}{2}\right)\right|+\frac{x_{i}}{12}\left(\frac{x_{i}+x_{i+1}}{2}\right)^{\alpha-1}\left|h^{\prime}\left(\frac{x_{i}+x_{i+1}}{2}\right)\right| \\
+ & \frac{x_{i+1}^{\alpha}}{6}\left|h^{\prime}\left(x_{i+1}\right)\right|-\frac{1}{6}\left(\frac{x_{i}+x_{i+1}}{2}\right)^{\alpha}\left|h^{\prime}\left(x_{i+1}\right)\right| \\
+ & \frac{x_{i+1}^{\alpha}}{3} \mid h^{\prime}\left(\frac{x_{i}+x_{i+1}}{2}\left|-\frac{1}{3}\left(\frac{x_{i}+x_{i+1}}{2}\right)^{\alpha}\right| h^{\prime}\left(\frac{x_{i}+x_{i+1}}{2}\right) \mid\right] \\
- & \frac{c}{8 \alpha}\left(x_{i+1}-x_{i}\right)^{3}\left[\frac{x_{i}^{\alpha}+\left(\frac{x_{i}+x_{i+1}}{2}\right)^{\alpha}-\frac{x_{i+1}^{\alpha}+\left(\frac{x_{i}+x_{i+1}}{2}\right)^{\alpha}}{20}}{12}\right. \\
+ & \left.\frac{x_{i+1}^{\alpha}-x_{i}^{\alpha}}{6}+\frac{x_{i}\left(\frac{x_{i}+x_{i+1}}{2}\right)^{\alpha-1}+x_{i}^{\alpha-1}\left(\frac{x_{i}+x_{i+1}}{2}\right)}{30}\right] .
\end{aligned}
$$

Proposition 2. Let $0 \leq x_{0}<x_{n}$ and $h:\left[x_{0}, x_{n}\right] \longrightarrow \mathbb{R}$ be an $\alpha$-fractional differentiable function for $\alpha \in(0,1]$. If $D_{\alpha}(h) \in L_{\alpha}^{1}\left(\left[x_{0}, x_{n}\right]\right)$ and $\left|h^{\prime}(x)\right|^{q}$ is strongly convex function with modulus $c>0$ for $q>1$ and $p^{-1}+q^{-1}=1$, then

$$
\begin{gathered}
\left|E_{\alpha}(h, P)\right| \leq \sum_{i=0}^{n-1} \frac{\left(x_{i+1}-x_{i}\right)}{2 \alpha}\left\{\left(S_{i, 1}(\alpha, p)\right)^{\frac{1}{p}}\left[\frac{\left|h^{\prime}\left(x_{i}\right)\right|^{q}+\left|h^{\prime}\left(\frac{x_{i}+x_{i+1}}{2}\right)\right|^{q}}{2}-\frac{c}{24}\left(x_{i+1}-x_{i}\right)^{2}\right]^{\frac{1}{q}}\right. \\
\left.+\left(S_{i, 2}(\alpha, p)\right)^{\frac{1}{p}}\left[\frac{\left|h^{\prime}\left(x_{i+1}\right)\right|^{q}+\left|h^{\prime}\left(\frac{x_{i}+x_{i+1}}{2}\right)\right|^{q}}{2}-\frac{c}{24}\left(x_{i+1}-x_{i}\right)^{2}\right]^{\frac{1}{q}}\right\} \\
\text { where } S_{i, 1}(\alpha, p)=\frac{2}{\left(x_{i+1}-x_{i}\right)} \int_{x_{i}}^{\frac{x_{i}+x_{i+1}}{2}}\left(t^{\alpha}-x_{i}^{\alpha}\right)^{p} d t \\
S_{i, 2}(\alpha, p)=\frac{2}{\left(x_{i+1}-x_{i}\right)} \int_{\frac{x_{i}+x_{i+1}}{2}}^{x_{i+1}}\left(x_{i+1}^{\alpha}-t^{\alpha}\right)^{p} d t .
\end{gathered}
$$


Proof. The proof is analogous to that of Proposition 1 only by using Corollary 4 of Theorem 4.

Proposition 3. Let $M>0,0 \leq x_{0}<x_{n}$ and $h:\left[x_{0}, x_{n}\right] \longrightarrow \mathbb{R}$ be an $\alpha$-fractional differentiable function for $\alpha \in(0,1]$. If $D_{\alpha}(h) \in L_{\alpha}^{1}\left(\left[x_{0}, x_{n}\right]\right)$ and $\left|h^{\prime}(x)\right|^{q}$ is strongly convex function with modulus $c>0$ for $q \geq 1$ and $\left|h^{\prime}(x)\right| \leq M, \forall x \in\left[x_{0}, x_{n}\right]$, then

$$
\begin{aligned}
\left|E_{\alpha}(h, P)\right| & \leq \frac{M}{2 \alpha} \sum_{i=0}^{n-1}\left(x_{i+1}-x_{i}\right)\left\{\left(C_{i, 1}(\alpha)\right)^{1-\frac{1}{q}}\left[C_{i, 2}(\alpha)+C_{i, 3}(\alpha)-\frac{c}{4}\left(x_{i+1}-x_{i}\right)^{2} E_{i, 1}(\alpha)\right]^{\frac{1}{q}}\right. \\
& \left.+\left(D_{i, 1}(\alpha)\right)^{1-\frac{1}{q}}\left[D_{i, 2}(\alpha)+D_{i, 3}(\alpha)-\frac{c}{4}\left(x_{i+1}-x_{i}\right)^{2} E_{i, 2}(\alpha)\right]^{\frac{1}{q}}\right\}
\end{aligned}
$$


where $C_{i, 1}(\alpha)=\frac{\left(\frac{x_{i}+x_{i+1}}{2}\right)^{\alpha+1}-x_{i}^{\alpha+1}}{(\alpha+1)\left(\left(\frac{x_{i}+x_{i+1}}{2}\right)-x_{i}\right)}-x_{i}^{\alpha}$,

$$
\begin{aligned}
& D_{i, 1}(\alpha)=x_{i+1}^{\alpha}-\frac{\left(\frac{x_{i}+x_{i+1}}{2}\right)^{\alpha+1}-x_{i+1}^{\alpha+1}}{(\alpha+1)\left(x_{i+1}-\left(\frac{x_{i}+x_{i+1}}{2}\right)\right)} \text {, } \\
& C_{i, 2}(\alpha)=-\frac{x_{i}^{\alpha+1}}{(\alpha+1)\left(\left(\frac{x_{i}+x_{i+1}}{2}\right)-x_{i}\right)} \frac{(\alpha+2)\left(\left(\frac{x_{i}+x_{i+1}}{2}\right)-x_{i}\right)+x_{i}}{(\alpha+2)\left(\left(\frac{x_{i}+x_{i+1}}{2}\right)-x_{i}\right)} \\
& +\frac{\left(\frac{x_{i}+x_{i+1}}{2}\right)^{\alpha+2}}{(\alpha+1)\left(\left(\frac{x_{i}+x_{i+1}}{2}\right)-x_{i}\right)^{2}(\alpha+2)}-\frac{x_{i}^{\alpha}}{2}, \\
& D_{i, 2}(\alpha)=\frac{x_{i+1}^{\alpha}}{2}+\frac{x_{i+1}^{\alpha+1}}{(\alpha+1)\left(x_{i+1}-\left(\frac{x_{i}+x_{i+1}}{2}\right)\right)} \frac{(\alpha+2)\left(x_{i+1}-\left(\frac{x_{i}+x_{i+1}}{2}\right)\right)+x_{i+1}}{(\alpha+2)\left(x_{i+1}-\left(\frac{x_{i}+x_{i+1}}{2}\right)\right)} \\
& -\frac{\left(\frac{x_{i}+x_{i+1}}{2}\right)^{\alpha+2}}{(\alpha+1)\left(x_{i+1}-\left(\frac{x_{i}+x_{i+1}}{2}\right)\right)^{2}(\alpha+2)} \\
& C_{i, 3}(\alpha)=\frac{\left(\frac{x_{i}+x_{i+1}}{2}\right)^{\alpha+1}}{(\alpha+1)\left(\left(\frac{x_{i}+x_{i+1}}{2}\right)-x_{i}\right)} \frac{(\alpha+2)\left(\left(\frac{x_{i}+x_{i+1}}{2}\right)-x_{i}\right)-\left(\frac{x_{i}+x_{i+1}}{2}\right)}{(\alpha+2)\left(\left(\frac{x_{i}+x_{i+1}}{2}\right)-x_{i}\right)} \\
& +\frac{x_{i}^{\alpha+2}}{(\alpha+1)\left(\left(\frac{x_{i}+x_{i+1}}{2}\right)-x_{i}\right)^{2}(\alpha+2)}-\frac{x_{i}^{\alpha}}{2} \text {, } \\
& D_{i, 3}(\alpha)=\frac{x_{i+1}^{\alpha}}{2}-\frac{\left(\frac{x_{i}+x_{i+1}}{2}\right)^{\alpha+1}}{(\alpha+1)\left(x_{i+1}-\left(\frac{x_{i}+x_{i+1}}{2}\right)\right)} \frac{(\alpha+2)\left(x_{i+1}-\left(\frac{x_{i}+x_{i+1}}{2}\right)\right)-\left(\frac{x_{i}+x_{i+1}}{2}\right)}{(\alpha+2)\left(x_{i+1}-\left(\frac{x_{i}+x_{i+1}}{2}\right)\right)} \\
& -\frac{x_{i+1}^{\alpha+2}}{(\alpha+1)\left(x_{i+1}-\left(\frac{x_{i}+x_{i+1}}{2}\right)\right)^{2}(\alpha+2)}, \\
& E_{i, 1}(\alpha)=\frac{1}{\left(\left(\frac{x_{i}+x_{i+1}}{2}\right)-x_{i}\right)^{3}} \\
& \times\left[\frac{\left(\frac{x_{i}+x_{i+1}}{2}\right)}{\alpha+2}\left(\left(\frac{x_{i}+x_{i+1}}{2}\right)^{\alpha+2}-x_{i}^{\alpha+2}\right)-\frac{x_{i}\left(\frac{x_{i}+x_{i+1}}{2}\right)}{\alpha+1}\left(\left(\frac{x_{i}+x_{i+1}}{2}\right)^{\alpha+1}-x_{i}^{\alpha+1}\right)\right. \\
& \left.-\frac{1}{\alpha+3}\left(\left(\frac{x_{i}+x_{i+1}}{2}\right)^{\alpha+3}-x_{i}^{\alpha+3}\right)-\frac{x_{i}}{\alpha+2}\left(\left(\frac{x_{i}+x_{i+1}}{2}\right)^{\alpha+2}-x_{i}^{\alpha+2}\right)\right]-\frac{x_{i}^{\alpha}}{6} \text {, }
\end{aligned}
$$




$$
\begin{aligned}
E_{i, 2}(\alpha) & =\frac{x_{i+1}^{\alpha}}{6}-\frac{1}{\left(x_{i+1}-\left(\frac{x_{i}+x_{i+1}}{2}\right)\right)^{3}} \\
& \times\left[\frac{x_{i+1}}{\alpha+2}\left(x_{i+1}^{\alpha+2}-\left(\frac{x_{i}+x_{i+1}}{2}\right)^{\alpha+2}\right)-\frac{1}{\alpha+3}\left(x_{i+1}^{\alpha+3}-\left(\frac{x_{i}+x_{i+1}}{2}\right)^{\alpha+3}\right)\right. \\
& \left.-\frac{x_{i+1}\left(\frac{x_{i}+x_{i+1}}{2}\right)}{\alpha+1}\left(x_{i+1}^{\alpha+1}-\left(\frac{x_{i}+x_{i+1}}{2}\right)^{\alpha+1}\right)+\frac{\left(\frac{x_{i}+x_{i+1}}{2}\right)}{\alpha+2}\left(x_{i+1}^{\alpha+2}-\left(\frac{x_{i}+x_{i+1}}{2}\right)^{\alpha+2}\right)\right] .
\end{aligned}
$$

Proof. The proof is analogous to that of Proposition 1 only by using Corollary 6 of Theorem 5.

Proposition 4. Let $0 \leq x_{0}<x_{n}$ and $h:\left[x_{0}, x_{n}\right] \longrightarrow \mathbb{R}$ be an $\alpha$-fractional differentiable function for $\alpha \in(0,1]$. If $D_{\alpha}(h) \in L_{\alpha}^{1}\left(\left[x_{0}, x_{n}\right]\right)$ and $\left|h^{\prime}(x)\right|^{q}$ is strongly convex function with modulus $c>0$ and $q \geq 1$, then

$$
\begin{aligned}
\left|E_{\alpha}(h, P)\right| & \leq \sum_{i=0}^{n-1} \frac{\left(x_{i+1}-x_{i}\right)}{2 \alpha} \\
& \times\left\{\left(L_{i, 1}(\alpha)\right)^{1-\frac{1}{q}}\left[\left|h^{\prime}\left(x_{i}\right)\right|^{q} M_{i, 1}(\alpha)+\left|h^{\prime}\left(\frac{x_{i}+x_{i+1}}{2}\right)\right|^{q} N_{i, 1}(\alpha)-\frac{c}{4}\left(x_{i+1}-x_{i}\right)^{2} P_{i, 1}(\alpha)\right]^{\frac{1}{q}}\right. \\
& \left.+\left(L_{i, 2}(\alpha)\right)^{1-\frac{1}{q}}\left[\left|h^{\prime}\left(x_{i+1}\right)\right|^{q} M_{i, 2}(\alpha)+\left|h^{\prime}\left(\frac{x_{i}+x_{i+1}}{2}\right)\right|^{q} N_{i, 2}(\alpha)-\frac{c}{4}\left(x_{i+1}-x_{i}\right)^{2} P_{i, 2}(\alpha)\right]^{\frac{1}{q}}\right\},
\end{aligned}
$$




$$
\begin{aligned}
& \text { where } L_{i, 1}(\alpha)=\frac{\left(\frac{x_{i}+x_{i+1}}{2}\right)^{\alpha}+x_{i}^{\alpha}}{3}+\frac{x_{i}\left(\frac{x_{i}+x_{i+1}}{2}\right)^{\alpha-1}+\left(\frac{x_{i}+x_{i+1}}{2}\right) x_{i}^{\alpha-1}}{6}-x_{i}^{\alpha} \text {, } \\
& L_{i, 2}(\alpha)==\frac{x_{i+1}^{\alpha}-\left(\frac{x_{i}+x_{i+1}}{2}\right)^{\alpha}}{2}, \\
& M_{i, 1}(\alpha)=\frac{\left(\frac{x_{i}+x_{i+1}}{2}\right)^{\alpha}-15 x_{i}^{\alpha}+x_{i}\left(\frac{x_{i}+x_{i+1}}{2}\right)^{\alpha-1}+\left(\frac{x_{i}+x_{i+1}}{2}\right) x_{i}^{\alpha-1}}{12}, \\
& N_{i, 1}(\alpha)=\frac{3\left(\frac{x_{i}+x_{i+1}}{2}\right)^{\alpha}-5 x_{i}^{\alpha}+x_{i}\left(\frac{x_{i}+x_{i+1}}{2}\right)^{\alpha-1}+\left(\frac{x_{i}+x_{i+1}}{2}\right) x_{i}^{\alpha-1}}{12}, \\
& M_{i, 2}(\alpha)=\frac{x_{i+1}^{\alpha}-\left(\frac{x_{i}+x_{i+1}}{2}\right)^{\alpha}}{6}, \\
& N_{i, 2}(\alpha)=\frac{x_{i+1}^{\alpha}-\left(\frac{x_{i}+x_{i+1}}{2}\right)^{\alpha}}{3}, \\
& P_{i, 1}(\alpha)=\frac{x_{i}^{\alpha}+\left(\frac{x_{i}+x_{i+1}}{2}\right)^{\alpha}}{20}+\frac{x_{i}\left(\frac{x_{i}+x_{i+1}}{2}\right)^{\alpha-1}+\left(\frac{x_{i}+x_{i+1}}{2}\right) x_{i}^{\alpha-1}}{30}-\frac{x_{i}^{\alpha}}{6}, \\
& P_{i, 2}(\alpha)=\frac{x_{i+1}^{\alpha}}{6}-\frac{x_{i+1}^{\alpha}+\left(\frac{x_{i}+x_{i+1}}{2}\right)^{\alpha}}{12} .
\end{aligned}
$$

Proof. The proof is analogous to that of Proposition 1 only by using Corollary 9 of Theorem 6 .

\section{Conclusion}

In this paper, using the concept of strongly convex functions and one known identity, we found several Ostrowski type inequalities pertaining conformable fractional integrals. Also, we give some error estimations for the midpoint formula.

\section{REFERENCES}

[1] Adil Khan M., Begum S., Kurshid Y., Chu Y. M., Ostrowski type inequalities involving conformable fractional integrals, J. Inequal. Appl., 2018(70), (2018).

[2] Agarwal, R. P., Luo, M. J., Raina, R. K., On Ostrowski type inequalities, Fasc. Math., 204, 5-27, (2016).

[3] Ahmadmir, M., Ullah, R., Some inequalities of Ostrowski and Grüss type for triple integrals on time scales, Tamkang J. Math., 42(4), 415-426, (2011).

[4] Alomari, M., Darus, M., Dragomir, S. S., Cerone, P., Ostrowski type inequalities for functions whose derivatives are s-convex in the second sense, Appl. Math. Lett., 23, 1071-1076, (2010).

[5] Anderson, D. R., Taylor's formula and integral inequalities for conformable fractional derivatives, Contributions in Mathematics and Engineering, Springer, 25-44, (2016).

[6] Azócar, A., Giménez, J., Nikodem, K., Sánchez, J. L., On strongly midconvex functions, Opuscula Math., 31(1), 15-26, (2011).

[7] Dragomir, S. S., On the Ostrowski's integral inequality for mappings with bounded variation and applications, Math. Ineq. \& Appl., 1(2), (1998). 
[8] Dragomir, S. S., The Ostrowski integral inequality for Lipschitzian mappings and applications, Comput. Math. Appl., 38, 33-37, (1999).

[9] Dragomir, S. S., Some perturbed Ostrowski type inequalities for absolutely continuous functions (I), Acta Univ. M. Belii, Ser. Math., 23, 71-86, (2015).

[10] Dragomir, S. S., Ostrowski-type inequalities for Lebesgue integral: A survey of recent results, Aust. J. Math. Anal. Appl., 14(1), 1-287, (2017).

[11] Dragomir, S. S., Wang, S., An inequality of Ostrowski-Grüss type and its applications to the estimation of error bounds for some special means and for some numerical quadrature rules, Comput. Math. Appl., 13(11), 15-20, (1997).

[12] Dragomir, S. S., Wang, S., A new inequality of Ostrowski's type in $L_{1}$-norm and applications to some special means and to some numerical quadrature rules, Tamkang J. Math., 28, 239-244, (1997).

[13] Farid, G., Some new Ostrowski type inequalities via fractional integrals, Int. J. Anal. App., 14(1), 64-68, (2017).

[14] Jovanovič, M.V., A note on strongly convex and strongly quasiconvex functions, Math. Notes, 60(5), 778-779, (1996).

[15] Kashuri, A., Liko, R., Ostrowski type fractional integral inequalities for generalized $(s, m, \varphi)$-preinvex functions, Aust. J. Math. Anal. Appl., 13(1), 1-11, (2016).

[16] Kashuri, A., Liko, R., Generalizations of Hermite-Hadamard and Ostrowski type inequalities for $M T_{m}$ preinvex functions, Proyecciones, 36(1), 45-80, (2017).

[17] Khalil, R., Alhorani, M., Yousef, A., Sababheh, M., A new definition of fractional derivative, J. Comput. Appl. Math., 264, 65-70, (2014).

[18] Liu, Z., Some Ostrowski-Grüss type inequalities and applications, Comput. Math. Appl., 53, 73-79, (2007).

[19] Liu W., Wen W., Park J., Ostrowski type fractional integral inequalities for MT-convex functions, Miskolc Math. Notes, 16(1), 249-256, (2015).

[20] Matloka, M., Ostrowski type inequalities for functions whose derivatives are h-convex via fractional integrals, Journal of Scientific Research and Reports, 3(12), 1633-1641, (2014).

[21] Merentes, N., Nikodem, K., Remarks on strongly convex functions, Aequationes Math., 80, 193-199, (2010).

[22] Nikodem, K., Páles, Zs., Characterizations of inner product spaces by strongly convex functions, Banach J. Math. Anal., 5(1), 83-87, (2011).

[23] Özdemir, M. E., Kavurmac, H., Set, E., Ostrowski's type inequalities for $(\alpha, m)$-convex functions, Kyungpook Math. J., 50, 371-378, (2010).

[24] Rafiq, A., Mir, N. A., Ahmad, F., Weighted Čebyšev-Ostrowski type inequalities, Applied Math. Mechanics (English Edition), 28(7), 901-906, (2007).

[25] Sarikaya, M. Z., On the Ostrowski type integral inequality, Acta Math. Univ. Comenianae, 79(1), 129-134, (2010).

[26] Tunç, M., Ostrowski type inequalities for functions whose derivatives are MT-convex, J. Comput. Anal. Appl., 17(4), 691-696, (2014).

[27] Ujević, N., Sharp inequalities of Simpson type and Ostrowski type, Comput. Math. Appl., 48, 145-151, (2004).

[28] Vial J. P., Strong and weak convexity of sets and functions, Math. Oper. Res., 8, 231-259, (1983).

[29] Yildiz, Ç., Özdemir, M. E., Sarikaya, M. Z., New generalizations of Ostrowski-like type inequalities for fractional integrals, Kyungpook Math. J., 56, 161-172, (2016).

[30] Zhongxue, L., On sharp inequalities of Simpson type and Ostrowski type in two independent variables, Comput. Math. Appl., 56, 2043-2047, (2008).

Department of Mathematics, Faculty of Technical Science, University Ismail Qemali, Vlora, Albania

E-mail address: artionkashuri@gmail.com 
Department of Mathematics, Faculty of Technical Science, University Ismail Qemali, Vlora, Albania

E-mail address: rozanaliko86@gmail.com 